\title{
Detection of fallow lands overgrowth from satellite images based on wavelet analysis
}

\author{
Larisa Evstratova ${ }^{1 *}$, Anton Antoshkin ${ }^{1}$ \\ ${ }^{1}$ State University of Land Use Planning, 105064, 15 Kazakova str., Moscow, Russian Federation
}

\begin{abstract}
To improve the efficiency of agricultural lands condition and use monitoring, method for detecting fallow lands overgrowth with tree and shrub vegetation using multi-temporal multispectral satellite images of high spatial resolution is proposed. The task of localization of overgrowth is solved on the basis of multi-temporal satellite images wavelet analysis. This technique allows to operationally and automatically detect areas of fallow lands overgrowth with tree and shrub vegetation. The proposed methodology was tested using satellite images obtained from Quickbird, GeoEye and WorldView satellites. For each image, a fourth-order wavelet decomposition was performed in terms of the Daubeshi wavelet functions. Comparing the different levels of the images wavelet transform, it is possible to divide the areas without tree and shrub vegetation, and where overgrowth was depicted. The method proposed by the authors for detecting changes based on wavelet analysis has shown that this method practically does not depend on the influence of shooting conditions and, accordingly, is more effective at detecting changes.
\end{abstract}

When solving the problem of effective involvement in the turnover of agricultural land, the question arises of determining the location and area of unused land plots subject to tree and shrub vegetation overgrowth. The most operational and reliable data for solving this problem are multispectral images obtained by space survey systems. However, automating the process of detecting changes based on multi-time satellite images is difficult task, in addition, visual analysis is often used when performing production work, which requires a lot of labor costs.

Thus, the search for new approaches for automated recognition of terrain objects images and identification of changes in multispectral satellite images at different times that would allow for prompt and effective the detecting of unused agricultural land and overgrowth long fallow is an urgent task.

Modern automated methods of decryption make it possible to recognize various objects with sufficient reliability. However, when analyzing multispectral images of different times, the spectral characteristics of the land lots may change, since they significantly depend on the shooting conditions and the object status. Therefore, algorithms in which the main decryption feature is the object spectral brightness cannot serve as a sufficient decryption feature $[1 ; 2]$. Many algorithms have been developed for detecting changes from

\footnotetext{
* Corresponding author: 1ge 21@mail.ru
} 
satellite images, but there is no universal method, as a rule, the algorithms are adapted for certain type of data (survey systems) and changes categories. In addition, different objects classes when recognized from satellite images often do not mutually exclude each other, which make it difficult to perform Change Detection procedures [1;3].

One of the solutions to the problem is the use of structural features of multispectral images of reference plots in satellite images. It is known that structural features are more stable, characterize the internal properties of object images directly related to its structure and practically does not change over time $[4 ; 5 ; 6]$, which means that algorithms based on these properties are more efficient than using spectral properties of images. Such features are obtained by Fourier transformation, wavelet transformation, by transformation using specially specified basis functions of various types (for example, spherical functions) [7; 8; 9].

The paper presents the results of researches of the proposed technique for the rapid detection of fallow lands overgrowth with woody and shrubby vegetation on multi-time multispectral satellite images based on the wavelet transform.

The purpose of this work is to assess the possibility of using wavelet analysis when processing multi-time images to localize the overgrowth of agricultural land plots with tree and shrub.

The paper provides an example of using wavelet functions in which decomposition is performed for different scales of the functions under study. When applying the wavelet decomposition, various levels of decompositions are analyzed in order to identify changes. In general, the wavelet transform of the function $f(t)$ can be written in the following form [9]:

$$
W[f(a, b)]=\frac{1}{\left|a^{1 / 2}\right|} \int_{-\infty}^{+\infty} f(t) \Psi\left(\frac{t-b}{a}\right) d t
$$

where $\Psi\left(\frac{t-b}{a}\right)$ - the basic wavelet function; $a$ - scaling parameter; $b$ - shift parameter; $\frac{1}{a^{1 / 2}}-$ the normalizing coefficient.

During decomposition, all images are "viewed" by the selected wavelet function and joint analysis of the levels and the function "location" in the image is performed. This makes it possible to localize changes in different-time images using the correlation coefficient calculated by the formula (2) as a criterion of compliance.

$$
k_{i j}=\frac{\sum_{x=i}^{N} \sum_{y=j}^{N}\left(P_{i}(x, y)_{-} P_{c p i}\right)\left(P_{j}(x, y)_{-} P_{c p j}\right)}{\left[\sum_{x=i}^{N} \sum_{y=j}^{N}\left(P_{i}(x, y)-P_{c p i}\right)^{2}\right]^{1 / 2}\left[\sum_{x=i}^{N} \sum_{y=j}^{N}\left(P_{j}(x, y)-P_{c p j}\right)^{2}\right]^{1 / 2}}
$$

where $k_{i j}$ - the correlation coefficient calculated between the levels $i$ and $j$ of the wavelet decomposition of the image $P$.

The research was conducted using high spatial resolution satellite images of Quickbird, GeoEye and WorldView. For the wavelet transform (in the discrete case), the numerical characteristics of the objects structural features in the image are matrices of coefficients with basic functions for each transformation scale level. The wavelet transformations were performed using Daubechies wavelet. Python 3.8 was used for software implementation and application of the wavelet transform for processing multispectral images.

Tree and shrub vegetation images differ from the surrounding vegetation on fallow land in brightness, tone, structure. The values of the wavelet decomposition coefficients 
depend on the values of the signal fluctuation, that is, in places of images where there are sharp differences in brightness values (the boundary of an object), larger values of the wavelet decomposition coefficients will be obtained than in homogeneous areas. Using this property, it is possible to localize the areas of the image, which depict land plots subject to tree and shrub vegetation overgrowth.

At the first stage of the research, the possibility of finding anomalies (tree and shrub vegetation overgrowth) on long fallow lands was determined using multispectral satellite images based on the obtained wavelet coefficients. The performed researches have shown that the Daubechies wavelet can be used as an indicator of the presence of changes in different-time images of the same area and used as a decoding feature of the image area.

In order to identify fallow lands overgrowth with tree and shrub vegetation, it is necessary to calculate the correlation coefficient between the corresponding levels of wavelet decomposition of different-time images according to formula 2. The correlation coefficient value will indicate the areas where changes have occurred.

At the second stage, researches of several images were carried out to identify changes in multi-time images. To do this, the correlation coefficient between the corresponding levels of the wavelet decomposition of images obtained on different dates was calculated. For the efficient operation of the algorithm, the image is divided into small fragments (the smallest areas) commensurate with the size of the smallest allocated overgrowth, and then a wavelet transform is performed for each smallest area (Fig. 1), this allows for further processing to use only the selected areas.
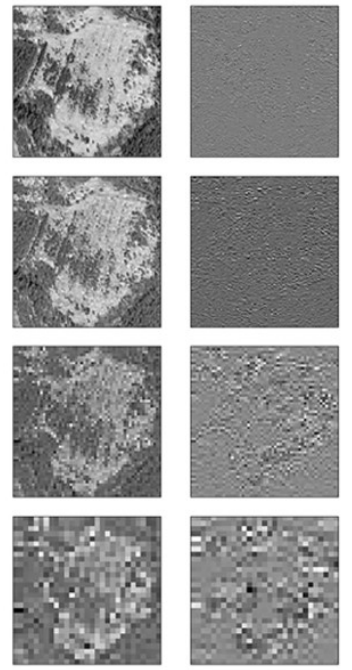

a)
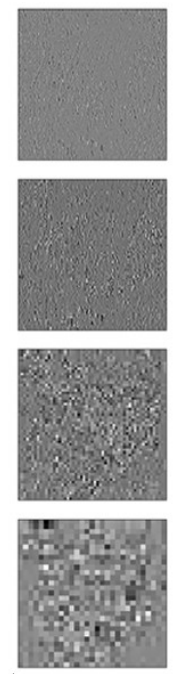
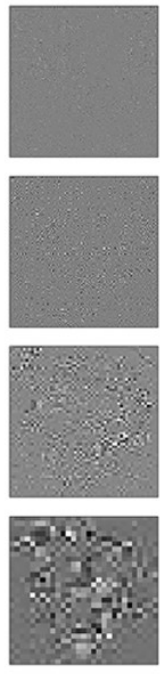
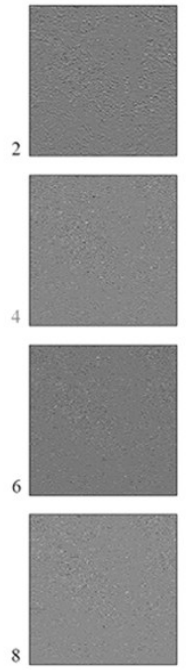
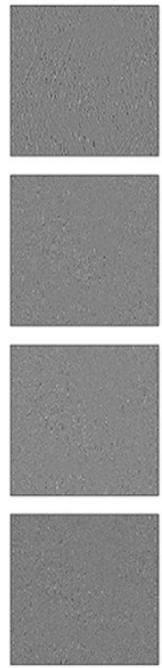

b)
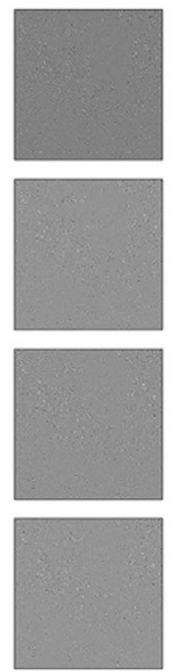

Fig1. Illustrations of image fragment Daubechies wavelet decomposition:

a) an image and its four level decomposition, b) scale transformation levels 2, 4, 6 and 8

The final stage of the technique of operational detection of unused land plots (fallow lands) subject to overgrowth by tree and shrub vegetation based on satellite images based on wavelet transformation is changes automatic vectorization in the images (Fig. 2).
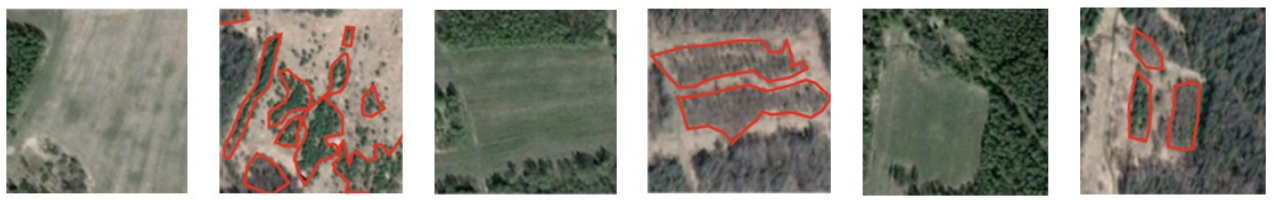

Fig. 2. Several images fragments as of 2009 and 2020 with detected overgrowth fallow by the 4th level wavelet decomposition 
Then the detected changes are combined with the cartographic material at an early date in order to update outdated data.

As a result of the performed researches, it is shown that the wavelet analysis can be used at the stage of preliminary processing of multispectral satellite images in order to localize areas with fallow lands overgrowth with tree and shrub vegetation. This technique makes it possible to automate as much as possible the process of fallow lands overgrowth preliminary detection with woody and shrubby vegetation based on multi-time high spatial resolution satellite images and also to increase the processing efficiency of satellite images obtained by various surveys space systems and under different shooting conditions of the same territory.

The practical value of the proposed approach lies in the fact that the algorithm implemented in the work makes it possible to improve numerical indicators when high spatial resolution images processing of on the example of localization of fallow lands overgrowth areas with tree and shrub vegetation, and can also be used in the agricultural lands monitoring system.

\section{References}

1. D. Lu, P. Mausel, E. Brondi'Zio, E. Moran Change detection techniques, International journal of Remote Sensing, 25, no 12, 2365-2407, (2003)

2. A. P. Guk, M. A. Altyntsev, L. G. Evstratova. Algorithms of multispectral aerospace image sequential analysis based on the use of structural-statistical approach for natural object decoding, in Proceedings of the All-Russian Conference Spatial Data Processing for Monitoring of Natural and Anthropogenic Processes, SDM 2019, 26-30 August 2019, Berdsk, Russia, 77-83, (2019). CEUR Workshop Proceedings ISSN 1613-0073, http://ceur-ws.org/Vol-2534/

3. P. Coppin, I. Jonckheere, K. Nackaerts, B. Muys Digital change detection methods in ecosystem monitoring: a review, International journal of Remote Sensing, 25, no.9, 1565-1596, (2004)

4. C. Blatter, "Wavelets-Eine Einführung," 2nd Edition, Vieweg, Wiesbaden, 2003.

5. William K. Pratt. Digital image processing. Wilely, $4^{\text {th }}$ ed, New York, (2007)

6. Gordienko A.S. The use of wavelet analysis in the processing of aerospace images. Scientific journal «Proceedings of the Higher Educational Institutions. Izvestia vuzov «Geodesy and aerophotosurveying», 2, 68-71, (2010)

7. Marc Antonini, Michel Barlaud, Pierre Mathieu, and Ingrid Daubechies. Image coding using wavelet transform. IEEE Transactions on image processing, 1, no. 2, 205-220, (1992)

8. Stephane G Mallat. A theory for multiresolution signal decomposition: the wavelet representation. IEEE transactions on pattern analysis and machine intelligence, 11, no.7, 674-693, (1989)

9. Christopher Torrence and Gilbert P Compo. A practical guide to wavelet analysis. Bulletin of the American Meteorological society, 79, no.1, 61-78, (1998) 